\title{
Fabrication d'aliment du bétail issue de la cueillette de gousses de Piliostigma reticulatum : une opportunité pour les femmes rurales burkinabé
}

\author{
Madjelia Cangré Ebou Dao ${ }^{1, *}$, Martine Kone ${ }^{1}$ et Jacques Somda ${ }^{2}$ \\ ${ }^{1}$ Centre national de la recherche scientifique et technologique, 03 BP 7047, Ouagadougou, Burkina Faso \\ ${ }^{2}$ Union internationale pour la conservation de la nature (UICN), Ouagadougou, Burkina Faso
}

\begin{abstract}
Résumé - Les gousses de l'arbre fourrager de Piliostigma reticulatum sont très utilisées au Burkina Faso. Comme peu de données existent sur cette activité, une enquête a été conduite en zone nord-soudanienne auprès de 90 personnes. Les résultats montrent que la collecte et la transformation des gousses sont menées par des femmes ménagères non instruites, âgées de 20 à 60 ans. La quantité moyenne collectée par les jeunes (20-35 ans), les moins âgées (36-50 ans) et les plus âgées (51-60 ans) est respectivement de 20, 5 et 10 t par femme. La vente de la farine leur procure un revenu moyen de, respectivement, 40000,14000 et 32000 FCFA par femme $(1000 \mathrm{FCFA}=1,5 €)$. Les moins de 35 ans s'intéressent particulièrement à la vente ; leurs contraintes sont d'ordre commercial (débouchés, organisation de la filière), tandis que les plus âgées ont indiqué des contraintes de gestion et de disponibilité des gousses (coupe des arbres, feux de brousses, animaux...). Les principales solutions préconisées par les jeunes sont la recherche de nouveaux débouchés pour la farine, tandis que pour les plus âgées ce sont la lutte contre les coupes d'arbres et la divagation des animaux. L'étude a montré que cette activité génère des revenus pour les jeunes femmes rurales démunies et fournit aux éleveurs des compléments alimentaires fourragers en saison sèche.
\end{abstract}

Mots clés : filière / gousse / Piliostigma / commercialisation / aliment du bétail

\begin{abstract}
Livestock feed manufacturing from gathering pods of Piliostigma reticulatum: an opportunity for rural women in Burkina Faso. Piliostigma reticulatum pods are increasingly exploited and marketed in Burkina Faso. As little knowledge exists about this activity, a survey was conducted in 2009 in the northern Sudan zone with 90 persons. Results show that gathering and processing pods is run by illiterate and poor women of 20- to 60 -year-old. An average of 20, five and 10 tons is gathered by 20-35-year-old, 36-50-year-old and 51-60-year-old women, respectively. The collected pods are crushed into flour with the help of children. Its sale provides an average income of 40,000, 14,000 and 32,000 FCFA per woman, respectively $(1000 \mathrm{FCFA}=1.5 €)$. The Chi-squared test at $5 \%$ revealed that young women under 35 are most interested in sale and are concerned with trade (opportunities, value chain), while oldest indicate weaknesses in management and availability of pods (uncontrolled cutting of trees, bush fires, stray animals...). Looking for new markets is the main solution proposed by young- and medium-aged women while older women want to fight against anarchic tree exploitation and livestock stray and graze. The results of this study demonstrate that gathering and processing P. reticulatum pods contribute to fight against poverty among rural women, decrease the unemployment rate and provide forage for animal breeders during the dry season.
\end{abstract}

Keywords: fodder / pod industry / exploited potential / commercialization / flour

\section{Introduction}

Les populations du Burkina Faso ont, depuis des siècles, utilisé les produits forestiers non ligneux (PFNL) que sont les fruits, les fleurs, les feuilles, l'écorce et les racines pour leur subsistance, mais aussi pour la commercialisation pour obtenir

\footnotetext{
$\overline{* \text { Auteur de correspondance }}$ : dao.ebou@gmail.com
}

des revenus et améliorer leur santé (Hill et al., 2007 ; Ouedraogo et al., 2013). Le rôle fourrager des PFNL a pris de l'ampleur ces dernières décennies à cause des besoins alimentaires croissants du bétail et de la faible disponibilité des fourrages herbacés en saison sèche (MRA, 2011). À ce titre, les gousses de l'arbre Piliostigma reticulatum Lam. sont utilisées pour l'alimentation des animaux. En zone nord-soudanienne du Burkina Faso, les gousses de P. reticulatum sont exploitées par les populations rurales (CIFOR, 2006 ; Sanou et al., 2010). 
Cette zone, caractérisée par une densité moyenne de 82 habitants $/ \mathrm{km}^{2}$ (pays : 47 habitants $/ \mathrm{km}^{2}$ ) et une croissance démographique estimée en 2012 à $2,4 \%$, présente plus de $51 \%$ de femmes. Celles-ci sont très vulnérables (INSD, 2008) et sont engagées dans la collecte des gousses.

Si à l'origine, les gousses collectées dans les formations naturelles étaient destinées à l'autoconsommation, la situation a progressivement changé. En effet, d'autres usages, notamment commerciaux, ont fait leur apparition ces dernières années, faisant de $P$. reticulatum une source d'alimentation du bétail et donc une source de revenus monétaires pour les populations rurales (CIFOR, 2006). Si la contribution des ressources végétales sauvages comme aliments d'appoint pour le bétail est très appréciée, force est de constater qu'elle est peu mesurée (CIFOR, 2006). La plupart des travaux portant sur les PFNL fourragers sont des études descriptives, botaniques et ethnobotaniques, ou d'évaluation de valeurs nutritives et bromatologiques (Dao et al., 2012 ; Ickowicz et al., 2005 ; Sanou et al., 2010 ; Yélémou et al., 2007 ; Zoundi et al., 1996). Le manque d'informations sur la transformation, les perspectives commerciales, le circuit de distribution et les potentiels exploités limite la prise en compte de cette activité par les décideurs et par conséquent freine les possibilités de valorisation et de gestion durable de cette ressource. La connaissance du degré d'exploitation des ressources et des difficultés rencontrées par les acteurs constitue un enjeu pour leur gestion durable. L'objectif de cette étude est de déterminer les quantités de gousses de $P$. reticulatum produites et exploitées par les populations en zone nord-soudanienne du Burkina Faso, d'identifier les atouts et les contraintes, et les solutions envisagées pour une exploitation durable de la ressource.

\section{Matériel et méthodes}

\subsection{Zone d'étude}

L'étude a été réalisée dans le village de Budtenga (12²9/ N, $1^{\circ} 15$, W) à $40 \mathrm{~km}$ de Ouagadougou, au Burkina Faso. Situé en zone nord-soudanienne, il se caractérise par une pluviométrie moyenne annuelle de 600 à $900 \mathrm{~mm}$ et une température moyenne annuelle de $29,5^{\circ} \mathrm{C}$. La végétation de type soudano-sahélien est clairsemée et caractérisée par la présence d'une strate ligneuse dominée par Vitellaria paradoxa, Acacia albida, Acacia seyal, Combretum aculeatum, Ziziphus mauritiana, Lannea microcarpa et Piliostigma reticulatum. La strate herbacée est dominée par des graminées annuelles telles que Loudetia togoensis et Andropogon pseudapricus. La population est rurale et se compose majoritairement de Moosi et secondairement de Bissa et de Peuls (INSD, 2008). L'activité principale est l'agriculture et secondairement l'élevage, l'exploitation des produits forestiers ligneux et non ligneux et leur commercialisation. Le cheptel est composé de ruminants et de monogastriques élevés suivant un système extensif. Mais, dans le village de Budtenga, existent aussi des fermes d'élevage orientées vers la production laitière, ainsi que des fermes d'élevage semi-intensif de volaille, ruminants et porcs pour la production de viande. Comme dans beaucoup d'élevages périurbains du Burkina Faso, les élevages semi-intensifs de Budtenga offrent des opportunités de valorisation des PFNL, parmi lesquels les gousses de $P$. reticulatum occupent une place importance. Le choix de ce site est donc justifié par :
- la dynamique de développement de l'élevage semi-intensif utilisant les gousses de $P$. reticulatum;

- la présence d'un peuplement de $P$. reticulatum ;

- l'implication des femmes pauvres dans la récolte des gousses de $P$. reticulatum;

- la représentativité de Budtenga en termes de dynamique de valorisation commerciale des gousses de $P$. reticulatum.

\subsection{Collecte et analyse des données}

Une enquête a été menée de juin 2008 à avril 2009 auprès d'un échantillon aléatoire de 90 femmes, soit deux tiers des collectrices de gousses de Budtenga ; elle a été réalisée sur la base de questionnaires concernant : les caractéristiques sociodémographiques, l'estimation des quantités de gousses exploitées, leurs usages, les quantités commercialisées et le circuit de vente, les atouts/contraintes de cette activité et les solutions envisagées.

Les caractéristiques sociodémographiques collectées portaient sur le nom et le prénom des personnes interrogées, l'âge, le sexe, le niveau d'éducation, la situation matrimoniale, les activités principales menées, l'appartenance à une organisation coopérative et le nombre d'années d'expérience dans la collecte des gousses. Une estimation de la production des arbres a été réalisée à partir de trois parcelles de 0,25 ha $(50 \times 50 \mathrm{~m})$ chacune. Un comptage systématique du nombre de pieds de $P$. reticulatum a été réalisé, puis la production a été estimée. Pour estimer la production, nous avons déterminé la quantité moyenne de gousses par arbre, le nombre de pieds à l'hectare et le nombre de pieds portant des fruits au moment de l'étude. La production moyenne obtenue par arbre est rapportée au pourcentage d'arbres portant les fruits pour avoir la production totale par hectare. Les quantités de gousses collectées, transformées et les différents usages sont obtenus par enquête. Les données collectées ont été analysées par la statistique descriptive (pourcentage, moyenne, écart-type). Elles ont été rapportées à trois classes d'âge : jeunes (20-35 ans), moins âgées (36-50 ans) et plus âgées (51-60 ans). Une matrice « swot» a permis de classifier les contraintes, les atouts, les opportunités et les menaces de l'activité de collecte (Keca et al., 2013). Le test de $\mathrm{Khi}^{2}$ au seuil de $5 \%$ a été réalisé à l'aide du logiciel SPSS version 19 pour déterminer les relations entre les classes d'âge et les variables de production et d'exploitation de gousses. La quantité valorisée par l'activité a été estimée à partir de la quantité de gousses collectée par les femmes rapportée à la quantité totale produite par hectare. Afin d'ordonner les solutions envisagées par les personnes interrogées lors de l'enquête, nous nous sommes basés sur la formule suivante de Ayuk (1997) :

$$
R W_{i}=\sum_{j=1}^{3}\left(W_{j}-F_{j}\right)
$$

où :

- $W_{\mathrm{j}}$ représente l'importance attribuée à la solution donnée par la $j$ eersonne enquêtée ;

- $F_{\mathrm{j}}$ représente la fréquence des solutions données au sein des 90 personnes enquêtées ;

- $R W$ est l'importance relative de la $i^{\mathrm{e}}$ solution donnée.

Pour chaque solution, $R W$ a été calculé. Les $R W_{\mathrm{i}}$ ont ensuite été comparées pour arriver à un classement final. 
Tableau 1. Quantité de gousses collectées par classe d'âge.

Table 1. Quantity of pods collected per group of age.

\begin{tabular}{lccccc}
\hline $\begin{array}{l}\text { Classe de } \\
\text { distribution }\end{array}$ & $\begin{array}{l}\text { Nombre de femmes par } \\
\text { classe de distribution }\end{array}$ & $\begin{array}{l}\text { Quantité moyenne/ } \\
\text { collectrice }(\mathrm{kg})\end{array}$ & $\begin{array}{l}\text { Quantité totale } \\
\text { de gousses }(\mathrm{kg})\end{array}$ & $\begin{array}{l}\text { Quantité de farine } \\
\text { obtenue }(\mathrm{kg})\end{array}$ & $\begin{array}{l}\text { Part de la farine } \\
\text { transformée }(\%)\end{array}$ \\
\hline Classe 1 : 20-35 & 26 & $19907 \pm 511$ & 517600 & 64700 & 59 \\
Classe 2 : 36-50 & 55 & $4819 \pm 170$ & 265040 & 33130 & 30 \\
Classe 3 : 51-60 & 9 & $10844 \pm 140$ & 97600 & 12200 & 11 \\
Total & 90 & & 880240 & 110030 \\
\hline
\end{tabular}

On a attribué à la première solution jugée la plus importante 5 points, à la deuxième 3 points et à la troisième 1 point.

\section{Résultats}

\subsection{Potentiel de production et d'exploitation des gousses}

Le ramassage des gousses de $P$. reticulatum a lieu principalement de décembre à avril; c'est une activité essentiellement féminine, pratiquée par des ménagères âgées de 20 à 60 ans, en majorité analphabètes. Elles sont accompagnées pour la plupart de leurs enfants car ce sont eux qui grimpent aux arbres pour cueillir les gousses et les mères assurent le ramassage au sol. À chaque passage, toute la production mature de l'arbre est cueillie. Pendant cette collecte, en moyenne $17 \pm 8$ pieds de $P$. reticulatum sont visités par jour et par femme, avec une distance moyenne parcourue de $15 \pm 6 \mathrm{~km}$ par jour. L'accès à la ressource est libre et les femmes munies de paniers se déplacent pour collecter les gousses matures, aidées par leurs enfants. Selon l'enquête, les pieds fructifères sont rencontrés principalement dans les basfonds ou le long des milieux plus humides. Bien que l'âge des arbres récoltés n'ait pas pu être estimé, le peuplement de $P$. reticulatum de la zone d'étude présente une physionomie vieillissante. La production de $P$. reticulatum a été estimée en moyenne à $6 \pm 5,7 \mathrm{~kg}$ de gousses sèches par arbre, avec un nombre moyen d'arbres de $477 \pm 4 /$ ha et $43 \%$ d'arbres fructifères en 2008-2009. La production totale des arbres a été évaluée à $1230 \mathrm{~kg} / \mathrm{ha}$. La quantité récoltée par les femmes a été évaluée à plus de $850 \mathrm{t}$; elle est repartie de manière variable selon les classes d'âge (Tab. 1). Les classes d'âge jeunes et moins âgées ont été les plus nombreuses à pratiquer cette activité, mais ce sont les jeunes qui ont collecté la part la plus importante des gousses : $20 \mathrm{t}$ en moyenne par femme, contre $5 \mathrm{t}$ pour les 30-50 ans et $10 \mathrm{t}$ pour les plus âgées (Tab. 1). Cette différence s'explique par le fait que les jeunes femmes disposent de moyens de transport (vélos et charrettes) leur permettant d'aller plus loin et de visiter plus de pieds de $P$. reticulatum dans la journée. De plus, ce sont elles-mêmes qui collectent sans attendre l'assistance des enfants qui ne sont pas toujours disponibles tous les jours parce que certains sont scolarisés.

\subsection{Transformation des gousses et utilisations}

Les gousses collectées sont ensuite transformées. Le procédé de transformation des gousses consiste à les sécher au soleil puis à les concasser dans des mortiers en même temps que les graines. On obtient ainsi de la farine de gousse. Ce processus de transformation est fait par les mêmes femmes qui collectent, accompagnées des enfants (Fig. 1). Ces enfants, membres de la famille, ne sont pas rémunérés. Pour obtenir $1 \mathrm{~kg}$ de farine de gousses, il a fallu concasser $8 \mathrm{~kg}$ de gousses sèches. La quantité de gousses transformées a été estimée à environ 65,33 et $12 \mathrm{t}$ pour respectivement la classe d'âge de 20-35, de 36-50 et de 51-60 ans (Tab. 2). Les enquêtes ont révélé quatre utilisations de la farine obtenue. Le premier usage, le plus important en quantité, a été la commercialisation : $69 \%$ de la quantité totale ; puis viennent l'autoconsommation (18\%), les dons ou les cadeaux aux membres de la famille ou autres personnes proches ( $11 \%$ ) et les autres usages ( $2 \%$ ) (Tab. 2). Les résultats du test d'indépendance de $\mathrm{Khi}^{2}$, réalisé sur les utilisations de la farine en fonction des âges des femmes, ont montré une différence significative au seuil de $5 \%$ $\left(\mathrm{Khi}^{2}\right.$ calculé $\left.=13,49 ; \mathrm{dl}=6 ; p<0,05\right)$. Les objectifs sociaux tels que le renforcement des relations sociales à travers des dons de farine aux parents ou amis et l'autoconsommation sont l'apanage des plus âgées. Tandis que la vente concerne plus les jeunes.

\subsection{Commercialisation et circuit de vente}

La farine destinée à la vente est conditionnée en unités de $50 \mathrm{~kg}$ et/ou dans des plats (Fig. 2). En 2009, les actrices clés de la commercialisation de cette farine ont été les jeunes de 20 à 35 ans, qui ont vendu de l'ordre de $45 \mathrm{t}$, contre $23 \mathrm{t}$ pour les moins âgées et $8 \mathrm{t}$ pour les plus âgées (Tab. 2). La quantité moyenne de farine vendue par femme a été évaluée à 2 , 0,7 et $1,6 \mathrm{t}$, respectivement, pour les $20-35,36-50$ et $51-60$ ans (Tab. 2). Ces ventes ont généré en moyenne 40000,14000 et 32000 FCFA par femme $(1000 \mathrm{FCFA}=1,5 €)$, respectivement les jeunes, les moins âgées et les plus âgées. Ceci correspond à un revenu d'environ 1500000 FCFA engrangé par les femmes de Budtenga durant la saison. Les différences de revenus entre les classes d'âge peuvent s'expliquer par le fait que les jeunes s'adonnent à cette activité parce que leur attractivité sur le marché du travail est faible compte tenu de leur absence d'instruction. Dans la commune d'Ourgou-Manega englobant le site d'étude, les femmes âgées de 15-45 ans sont à $84 \%$ analphabètes (INSD, 2008). L'alternative qui se présente à elle est la cueillette de ressources telles que les gousses de Piliostigma. Il n'y a pas eu d'intermédiaires dans les ventes. Les lieux de vente sont les concessions familiales ou les marchés locaux du village. Les collectrices sont à la fois transformatrices et vendeuses. Les acheteurs, composés à $90 \%$ 

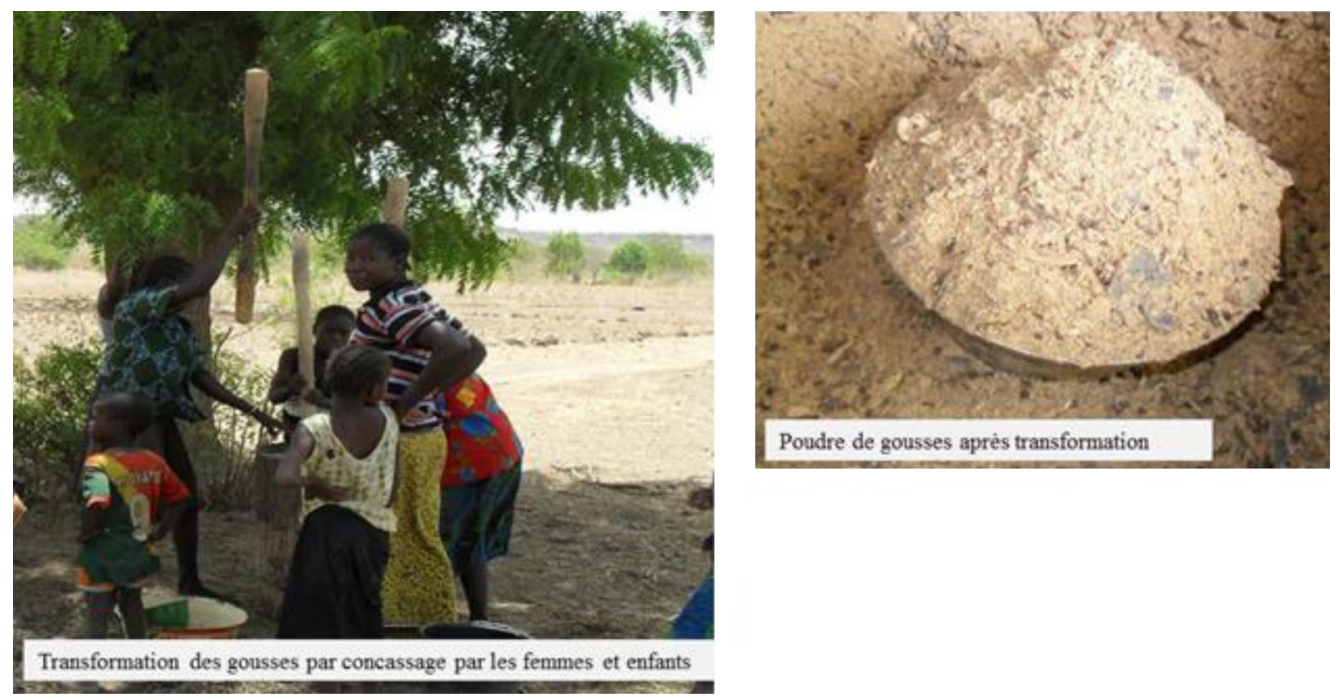

Fig. 1. Séance de concassage des gousses de Piliostigma reticulatum par les femmes et les enfants et poudre obtenue.

Fig. 1. Crushing session of Piliostigma reticulatum pods by women and children, and powder obtained. Photo MCE Dao, 2009.

Tableau 2. Effectifs de femmes $(n)$ et quantité de farine par usage et par classe d'âges.

Table 2. Number of women per group of age (n) and utilisations of pods flour.

\begin{tabular}{|c|c|c|c|c|c|c|}
\hline \multirow{2}{*}{ Usages de la farine } & \multicolumn{2}{|c|}{ Classe $1: 20-35$} & \multicolumn{2}{|c|}{ Classe $2: 36-50$} & \multicolumn{2}{|c|}{ Classe $3: 51-60$} \\
\hline & Quantité (kg) & $n$ & Quantité (kg) & $n$ & Quantité (kg) & $n$ \\
\hline Autoconsommation & 3050 & 2 & 15230 & 10 & 1520 & 1 \\
\hline Don & 2020 & 2 & 6050 & 6 & 4030 & 4 \\
\hline Autres & 360 & 1 & 1470 & 4 & 370 & 1 \\
\hline
\end{tabular}
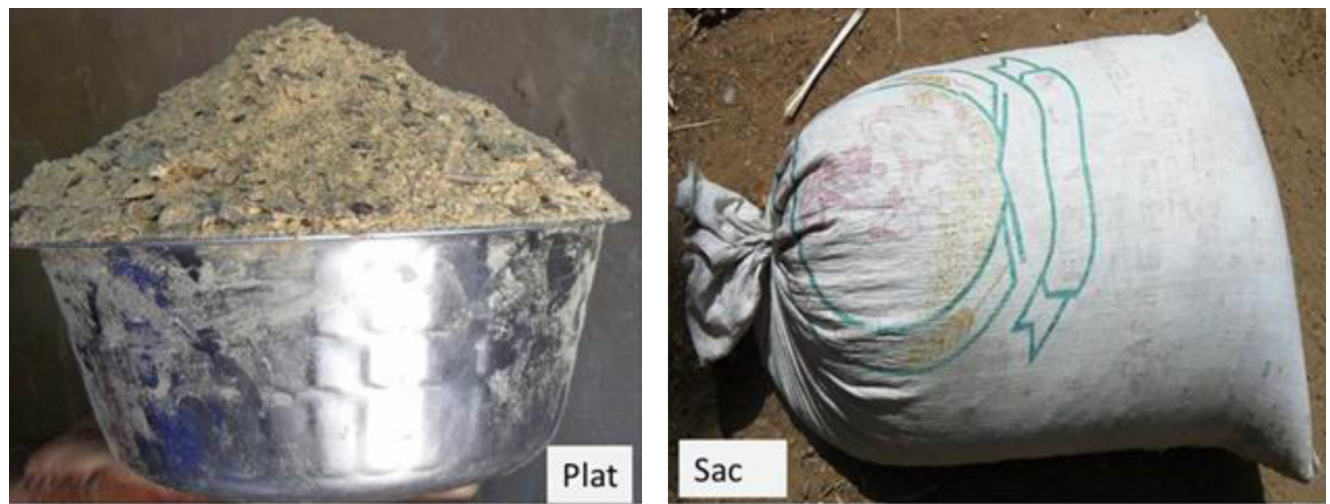

Fig. 2. Unités de vente de la poudre de gousses.

Fig. 2. Measurement units for selling pods powder. Photo MCE Dao, 2009.

d'éleveurs de porcins, caprins, ovins et bovins venus des zones périurbaines de la capitale Ouagadougou, sont les consommateurs directs du produit. Les $10 \%$ restant sont des éleveurs du village. Pour la fixation des prix, le marchandage est d'usage : le prix a été discuté dans plus de $80 \%$ des cas. Cinquante kilogramme de farine se vendent $1000 \mathrm{FCFA}$ en période de récolte et le tas (environ $2 \mathrm{~kg}$ ), 100 FCFA. Le prix est plutôt fixé par l'acheteur. Le prix de la farine de gousse $(20-50 \mathrm{FCFA} / \mathrm{kg})$ était inférieur à celui du maïs (150-200 FCFA/kg), du sojagraine $(250 \mathrm{FCFA} / \mathrm{kg})$, des aliments issus du mélange peaux + pulpe de mangue + son $(150 \mathrm{FCFA} / \mathrm{kg})$ qui sont tous des aliments localement utilisés dans l'alimentation des porcs et les 


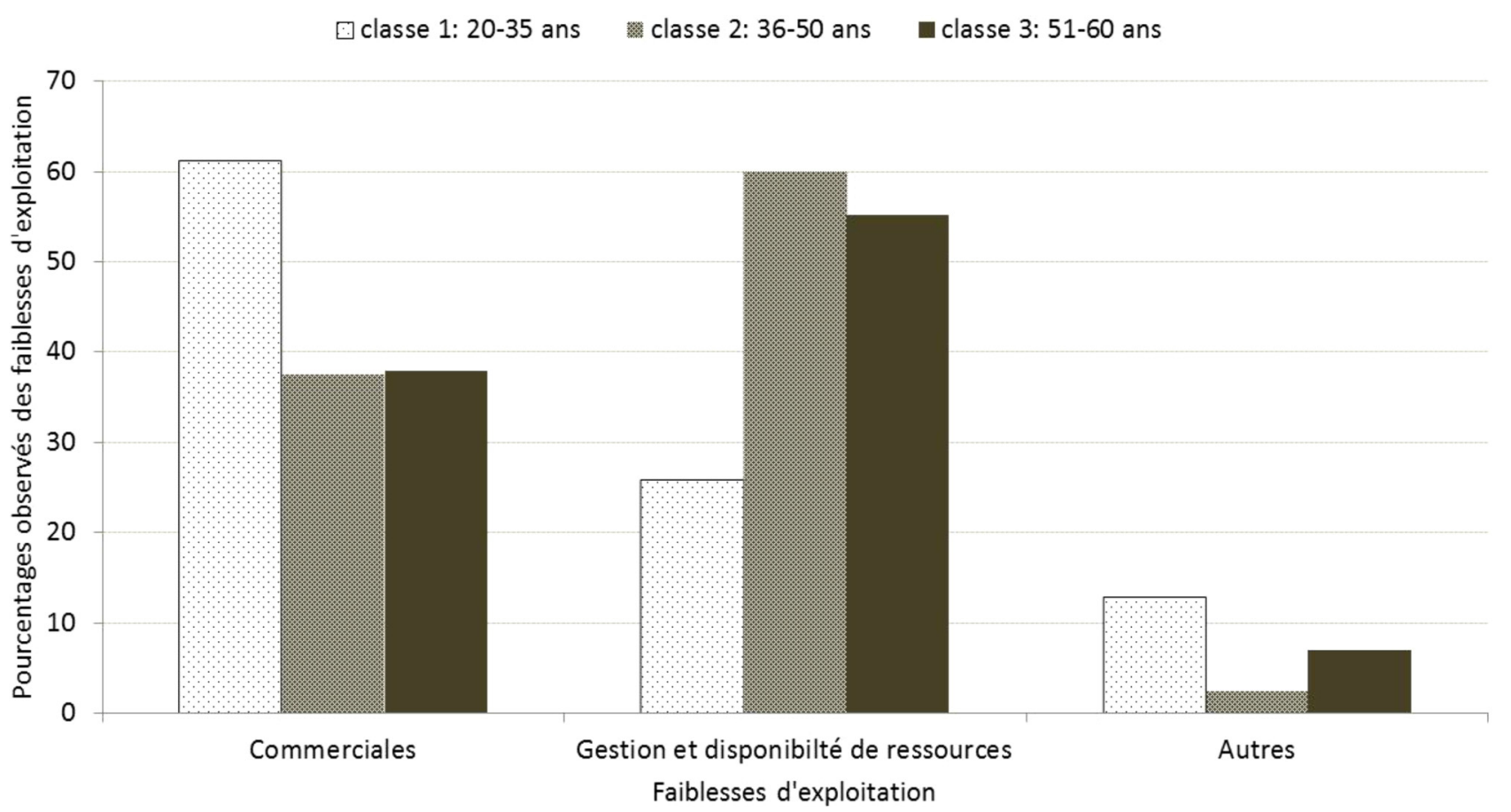

Fig. 3. Histogramme des contraintes selon les classes d'âge.

Fig. 3. Histogram of constraints by group of age.

Tableau 3. Solutions envisagées par les femmes.

Table 3. Alternatives solutions given by women.

\begin{tabular}{ll}
\hline$R W_{\mathrm{i}}$ & Solutions envisagées (échantillon =90) \\
\hline 16909 & $\begin{array}{l}\text { Recherche de nouveaux débouchés de vente des } \\
\text { produits }\end{array}$ \\
13832 & $\begin{array}{l}\text { Lutte contre l'ébranchage destructif et contre la coupe } \\
\text { des arbres }\end{array}$ \\
6750 & $\begin{array}{l}\text { Lutte contre les feux de brousses } \\
5698\end{array}$ \\
4876 & $\begin{array}{l}\text { Réalisation de plantations de Piliostigma reticulatum } \\
\text { Préservation de l'espèce lors des défrichements }\end{array}$ \\
12356 & $\begin{array}{l}\text { Organisation du marché (fixation des prix de vente) } \\
\text { Contrôles plus accentués de la police forestière }\end{array}$ \\
98 & Lutte contre la divagation des animaux
\end{tabular}

autres animaux (Organisation des Nations unies pour l'alimentation et l'agriculture, FAO, 2012 ; Kiendrebeogo et al., 2013).

\subsection{Contraintes et solutions envisagées par les femmes}

Les femmes rencontrent des contraintes liées principalement à la commercialisation des gousses et de la poudre et à la gestion et la disponibilité de la ressource (Fig. 3). Les contraintes commerciales sont l'insuffisance de nouveaux débouchés, la fixation des prix, l'inorganisation de la filière, l'absence de technologies appropriées de stockage, de conservation et de conditionnement occasionnant des pertes importantes de gousses. Elles ont été notées par environ $60 \%$ des 20-35 ans. La majorité des plus de 50 ans ont mentionné des contraintes liées à la ressource : faible production fruitière des arbres et attaques parasitaires des gousses.

Plusieurs solutions sont envisagées par les femmes pour une exploitation durable des gousses. Les principales mentionnées sont la recherche de nouveaux marchés, la lutte contre la coupe anarchique, contre l'ébranchage destructif et contre les feux de brousse, la préservation de l'espèce lors des défrichements, la plantation d'arbres (Tab. 3). La répartition des solutions proposées par classe d'âge montre que les jeunes et les moins âgées préconisent de rechercher de nouveaux débouchés tandis que les plus âgées suggèrent de lutter contre les coupes et la divagation des animaux (Fig. 4). Cependant, le test de $\mathrm{Khi}^{2}$ n'a pas montré de lien significatif entre classe d'âge et solutions proposées $\left(\mathrm{Khi}^{2}=8,93\right.$; $\mathrm{dl}=49 ; p>0,05)$.

\section{Discussion}

L'étude a permis d'identifier les atouts de l'activité, qui sont la création d'emplois en saison sèche pour une centaine de femmes et la génération de revenus moyens de 18000 FCFA par femme. La collecte requiert des techniques locales de transformation. La transformation en farine fournit des sousproduits locaux aux éleveurs de la localité et surtout des alentours de la capitale. Elle contribue aussi au renforcement des liens sociaux pour les plus âgées. Les dons se font généralement aux proches parents pour les aider dans l'alimentation des animaux (chèvres, moutons, bœufs ou porcs) pendant la période de soudure.

L'émergence de nouveaux marchés extérieurs pourrait concerner d'autres localités du Burkina Faso et constituer une opportunité pour les femmes. L'organisation des collectrices en 
M.C.E. Dao et al. : Cah. Agric. 2016, 25, 35002

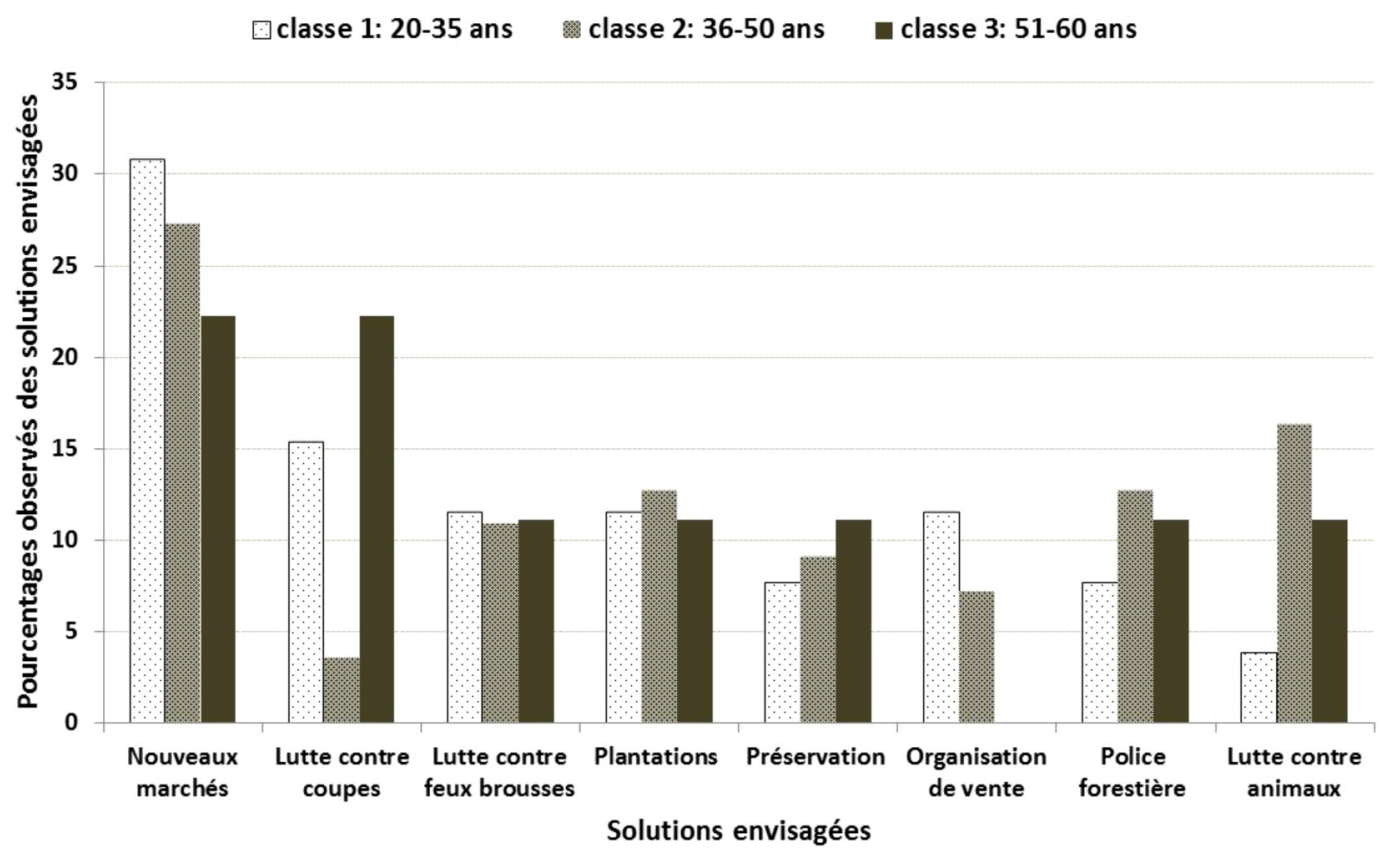

Fig. 4. Histogrammes des solutions envisagées selon les classes d'âge.

Fig. 4. Histograms of solutions given by group of age.

groupement pourrait permettre de viser des marchés urbains. L'existence d'une telle organisation permettrait également de mieux organiser la collecte des gousses et de protéger la ressource. D'autres travaux menées au Burkina Faso ont montré que lorsque les collectrices de noix de karité et les transformatrices en beurre de karité sont organisées, elles arrivent à améliorer la qualité de leurs produits, à mener des activités de promotion dans les foires, les expositions et les marchés, à conquérir de nouveaux marchés aux niveaux national et international, et à influencer les prix de vente de leurs produits (Badini et al., 2011 ; Elias et Carney, 2004 ; François et al., 2009 ; Saussey, 2006).

Les collectrices ont rencontré des difficultés dans la collecte et la valorisation des gousses. Les principales contraintes sont d'ordre organisationnel, commercial, de disponibilité et de gestion de la ressource. Elles déclarent : « Il n'y a pas beaucoup de gousses sur les arbres et nous sommes nombreuses à aller collecter. Si tu ne te lèves pas très tôt, tu n'as rien ». Ce qui est lié à la durabilité de la ressource - le pourcentage d'individus fructifères du peuplement (43\%) et la production moyenne (6 kg de gousses séchées par arbre) - sont insuffisants ; des travaux antérieurs ont noté que 30 à $40 \%$ des pieds de $P$. reticulatum de la zone nordsoudanienne ne produisent pas de gousses (Yélémou et al., 2007). L'absence de fructification peut s'expliquer par le caractère dioïque de $P$. reticulatum: pieds mâles non fructifères et pieds femelles fructifères (Dao et al., 2012). De plus, les gousses produites sont souvent de mauvaise qualité du fait d'attaques parasitaires (Dao et al., 2012 ; Yélémou et al., 2010). D'autres contraintes sont liées aux feux de brousse et aux pratiques de collectes; en effet, la technique de récolte par la coupe des branches fructifères est préjudiciable à la durabilité de la ressource. De telles pratiques occasionnent des dommages physiologiques et des maladies parasitaires ; elles seraient préjudiciables à plus de $20 \%$ des légumineuses d'Afrique (Oluoch et al., 2009).

Pour promouvoir une gestion durable de l'activité, les solutions proposées mettent l'accent sur des actions de protection et de valorisation (plantations et amélioration de la collecte et des circuits de vente). Ces actions sont similaires à celles proposées par les populations rurales du Nord Bénin, où $34 \%$ des exploitants des parcs à karité préconisent des actions de reboisement (Gnanglè et al., 2012). Cependant, ces solutions n'aboutiront que si des mesures d'accompagnement sont prises, comme la formation et l'encadrement technique et économique (marchés et filières).

\section{Conclusion}

Les arbres fourragers contribuent de façon significative aux revenus des femmes rurales pauvres. Les activités de collecte, de transformation et de commercialisation de la farine des gousses de $P$. reticulatum génèrent des revenus significatifs pour les jeunes femmes de Budtenga, où le taux de sous-emploi est l'un des plus élevés du pays. Le développement d'une foresterie communautaire serait nécessaire pour mieux exploiter les ressources forestières et approvisionner les centres urbains en fourrages. Cependant, le fonctionnement biologique de $P$. reticulatum reste à mieux étudier (causes biologiques de la faible production, entomofaune florale, techniques sylvicoles...) pour garantir la durabilité de l'activité. Les opportunités qu'offrent $P$. reticulatum comme complément fourrager en saison sèche doivent aussi être mieux connues des décideurs politiques, car, sans volonté politique, son exploitation risque d'être éphémère. 


\section{Références}

Ayuk ET. 1997. Adoption of agroforestry technology: the case of live hedges in the Central Plateau of Burkina Faso. Agric. Syst. 54: 189-206.

Badini Z, Kaboré M, Mheen-Sluijer J, Vellema S. 2011. Chaînes de valeur de la filière karité au Burkina Faso. Wageningen, VC4PD Research Paper No. 14: 1-45. https://www.wageningenur.nl/upload mm/7/2/c/ b20ed990-9828-4a90-8343-d6d0330c892d_VC4PDResearchPaper_ 14_Burkina.pdf.

CIFOR. 2006. Les gousses de Piliostigma sp., Ouagadougou, Burkina Faso, Ed. CIFOR-CNRST, 26 p.

Dao MCE, Diallo BO, Kaboré-Zoungrana C. 2012. Fruit and seed production in a natural population of a dioecious plant: P. reticulatum Hochst. Int. J. Biol. Chem. Sci. 6(1): 11-23.

Elias M, Carney J. 2004. La filière féminine du karité : productrices burkinabé, " éco-consommatrices » occidentales et commerce équitable. Cah. Geogr. Quebec 48: 71-88.

FAO, 2012. Secteur porcin Burkina Faso. Revues nationales de l'élevage de la division de la production et de la santé animales de la FAO. No 1. Rome. 83 p.

François M, Niculescu N, Badini Z, Diara M. 2009. Le beurre de karité au Burkina Faso: entre marché domestique et filières d'exportation. Cah. Agric. 18(4): 369-375.

Gnanglè PC, Yabi JA, Yegbemey NR, Kakaï RG, Sokpon N. 2012. Rentabilité économique des systèmes de production des parcs à karité dans le contexte de l'adaptation au changement climatique du Nord Benin. Afr. Crop Sci. J. 20(IS2): 589-602.

Hill T, Ouedraogo Y, Conditamde L, 2007. L'entreprise villageoise d'exploitation des arbres au Burkina Faso : appuyer le développement des petites entreprises dont l'activité est fondée sur les produits forestiers non ligneux. In: La foresterie à petite échelle. Unasylva 228(58). Rome (Italie): FAO. http://www.fao.org/docrep/010/ a1346f/a1346f00.htm.

Ickowicz A, Friot D, Guerin H. 2005. Acacia senegal, arbre fourrager sahélien. Bois Forets Trop. 284: 59-68.

INSD. 2008. Recensement général de la population et de l'habitation de 2006 du Burkina Faso. Résultats définitifs. Ed. Ministère de l'Economie et du Développement/Burkina Faso, 52 p.
Keca LJ, Keca N, Rekola M. 2013. Value chains of Serbia non-wood forest products. Int. Forestry Rev. 15(3): 315-335.

Kiendrebeogo T, Mopate LY, Ido G, Kaboré-Zoungrana CY. 2013. Procédés de production d'aliments non conventionnels pour porcs à base de déchets de mangues et détermination de leurs valeurs alimentaires au Burkina Faso. J. Appl. Biosci. 67: 5261-5270.

MRA. 2011. Deuxième Enquête Nationale sur les Effectifs du Cheptel (ENEC II). Projection des Résultats et Analyses (Tome II). Ed. Ministère des Ressources Animales, Ouagadougou Burkina Faso, $156 \mathrm{p}$.

Oluoch MO, Pichop GN, Silue D, Abukutsa-Onyango MO, Diouf M, Shackleton CM. 2009. Production and harvesting systems for African indigenous vegetables. In: Shackleton CM, Pasquini MW, Drescher AW, eds. African indigenous vegetables in urban agriculture, London (United Kingdom): Earthscan, pp. 145-175.

Ouedraogo M, Ouedraogo D, Thiombiano T, Hien M, Lykke AM. 2013. Dépendance économique aux produits forestiers non ligneux : cas des ménages riverains des forêts de Boulon et de Koflandé, au Sud-Ouest du Burkina Faso. J. Agric. Environ. Int. Dev. 107(1): 45-72.

Sanou S, Sawadogo L, Kaboré-Zoungrana CY. 2010. Amélioration de la valeur nutritionnelle des gousses de Piliostigma reticulatum (DC) Hochst dans l'alimentation du bétail en période de soudure. Int. J. Biol. Chem. Sci. 4(5): 1519-1528.

Saussey M. 2006. Les associations féminines de productrices de beurre de karité au Burkina Faso : enjeux de développement durable. In: York University ed. International Congress of the Social Sciences and Humanities, Annual meeting of the Canadian Association for studies in co-operation. Toronto, Canada, York University 30/05-2/06/2006.

Yélémou B, Bationo B, Yaméogo G, Millogo-Rasolodimby J. 2007. Gestion traditionnelle et usage de P. reticulatum (DC) Hochst., dans le Plateau central du Burkina Faso. Bois Foret Trop. 291(1): 55-65.

Zoundi SJ, Nianogo AJ, Sawadogo L. 1996. Utilisation de gousses de Piliostigma reticulatum (DC) Hochst et de feuilles de Cajanus Cajan (L.) Millsp. en combinaison avec l'urée pour l'engraissement de moutons Djallonké type mossi et du sud au Burkina. Tropicultura 4: 149-152.

Cite this article as: Dao MCE, Kone M, Somda J. 2016. Fabrication d'aliment du bétail issue de la cueillette de gousses de Piliostigma reticulatum : une opportunité pour les femmes rurales burkinabé. Cah. Agric. 25: 35002. 